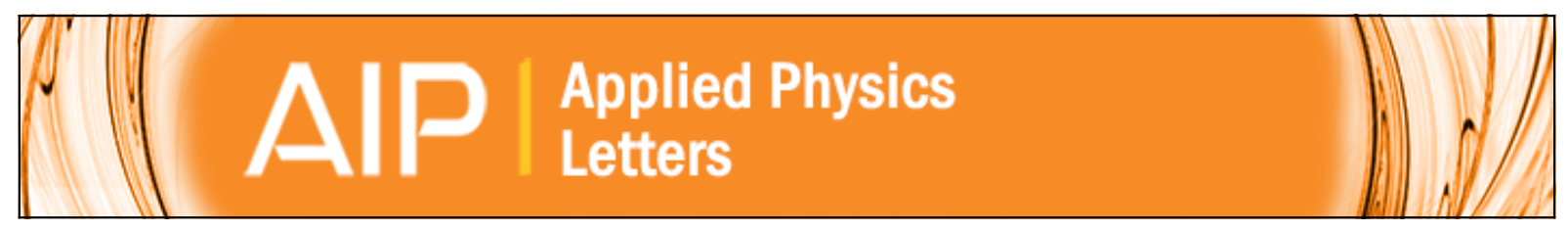

Patterned growth and cathodoluminescence of conical boron nitride nanorods

H. Z. Zhang, M. R. Phillips, J. D. Fitz Gerald, J. Yu, and Y. Chen

Citation: Applied Physics Letters 88, 093117 (2006); doi: 10.1063/1.2179144

View online: http://dx.doi.org/10.1063/1.2179144

View Table of Contents: http://scitation.aip.org/content/aip/journal/apl/88/9?ver=pdfcov

Published by the AIP Publishing

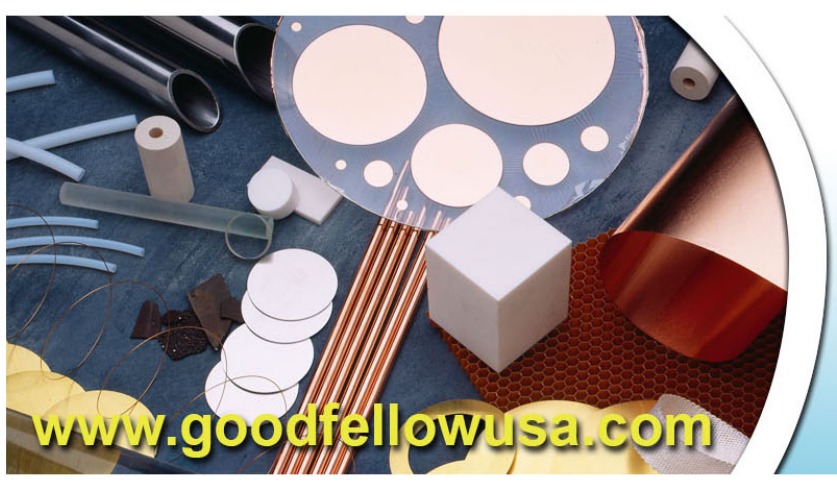

BODIFElIOU metals • ceramics • polymers composites $\cdot$ compounds $\cdot$ glasses

Save $5 \% \cdot$ Buy online 70,000 products $\cdot$ Fast shipping 


\title{
Patterned growth and cathodoluminescence of conical boron nitride nanorods
}

\author{
H. Z. Zhang ${ }^{\text {a) }}$ \\ Department of Electronic Materials Engineering, Research School of Physical Science and Engineering, \\ The Australian National University, Canberra, ACT 0200, Australia \\ M. R. Phillips \\ UTS Institute of Nanoscale Technology, University of Technology, Sydney, NSW 2007, Australia \\ J. D. Fitz Gerald \\ Research School of Earth Sciences, The Australian National University, Canberra, ACT 0200, Australia \\ J. Yu and Y. Chen \\ Department of Electrorric Materials Engineering, Research School of Physical Science and Engineering, \\ The Australian National University, Canberra, ACT 0200, Australia
}

(Received 21 June 2005; accepted 11 January 2006; published online 2 March 2006)

\begin{abstract}
We demonstrate a simple and effective approach for growing large-scale, high-density, and well-patterned conical boron nitride nanorods. A catalyst layer of $\mathrm{Fe}\left(\mathrm{NO}_{3}\right)_{3}$ was patterned on a silicon substrate by using a copper grid as a mask. The nanorods were grown via annealing milled boron carbide powders at $1300{ }^{\circ} \mathrm{C}$ in a flow of nitrogen gas. The as-grown nanorods exhibit uniform morphology and the catalyst pattern precisely defines the position of nanorod deposition. Cathodoluminescence (CL) spectra of the nanorods show two broad emission bands centered at 3.75 and $1.85 \mathrm{eV}$. Panchromatic CL images reveal clear patterned structure. (C) 2006 American Institute of Physics. [DOI: 10.1063/1.2179144]
\end{abstract}

Boron nitride is an isoelectronic analog of carbon and exhibits at least four polymorphic modifications: hexagonal $(h \mathrm{BN})$, rhombohedral $(r \mathrm{BN})$, zinc blende $(c \mathrm{BN})$, and wurtzitic $(w \mathrm{BN}){ }^{1}$ Because of their good mechanical, thermal, and chemical properties, $\mathrm{BN}$ powders and $\mathrm{BN}$ thin films have been widely used as refractory materials, lubricants, abrasive grains, and hard coatings. BN is also an electrical insulator, for example, the band gap of $c \mathrm{BN}$ is larger than $6.4 \mathrm{eV}$ while measurements of the band gap of $h \mathrm{BN}$ span a wide range from 3.6 to $7.1 \mathrm{eV}^{2,3}$ The large band gap makes boron nitride a promising candidate for deep-blue and UV applications. ${ }^{4}$ Its luminescence properties have been thus studied extensively, and a number of luminescence centers have been reported. ${ }^{3,5-9}$ An ultraviolet light-emitting diode of a cubic boron nitride $p n$ junction has been realized, ${ }^{10}$ and room-temperature ultraviolet lasing has been observed in an $h \mathrm{BN}$ single crystal. ${ }^{11}$ Quasi-one-dimensional boron nitride nanomaterials such as BN nanotubes, ${ }^{12} \mathrm{BN}$ nanocones, ${ }^{13} \mathrm{BN}$ helixes, ${ }^{14}$ and BN whiskers, ${ }^{15}$ have been investigated extensively. In contrast to carbon nanotubes, $\mathrm{BN}$ nanotubes have uniform electronic band gaps, independent of their diameters and chiralities. ${ }^{16}$ Quantum confinement effects in these lowdimensional materials can enhance their optical emission substantially by inducing an indirect-to-direct conversion of the optical transition. ${ }^{17,18}$ Therefore, one-dimensional BN nanomaterials are likely to find further applications in optoelectronics, but only a few reports exist about the luminescence properties of one-dimensional BN nanomaterials. ${ }^{15,19}$ Photoluminescence and cathodoluminescence of large BN whiskers consisting of nanofiber-interweaved network have been studied. $^{15,19}$ It is well known that impurities or defects can introduce acceptor or donor levels in the band gap of a

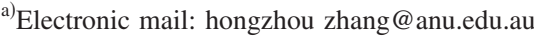

material, ${ }^{7,8}$ and one-dimensional $\mathrm{BN}$ nanomaterials with different structures and dimensions may well have different emission behaviors. Recently, conical boron nitride nanorods with a mean diameter of $60 \mathrm{~nm}$ have been synthesized by annealing ball-milled boron carbide. ${ }^{20}$ The unusual conical structure of these $\mathrm{BN}$ nanorods makes their luminescence properties an interesting topic. In addition, to our knowledge selective growth of one-dimensional BN nanomaterials, specifically patterned growth, has not been realized, which could become a hindrance for their further applications. In this letter, we report a simple and effective approach for patterned growth of the conical boron nitride nanorods and describe their cathodoluminescence properties.

Ball milling and annealing was used to grow the conical boron nitride nanorods, as reported elsewhere, milled boron carbide powders were annealed in a flowing nitrogen atmosphere. $^{20-22}$ To grow the nanorods selectively on a silicon substrate, i.e., patterned growth, a catalyst layer of $\mathrm{Fe}\left(\mathrm{NO}_{3}\right)_{3}$ was first deposited on the substrate as follows. A copper grid (3 $\mathrm{mm}$ in diameter, square mesh with a width of $50 \mu \mathrm{m})$ was clipped onto the polished surface of a silicon substrate. The temperature of the substrate and the grid was then kept at about $100{ }^{\circ} \mathrm{C}$. Several drops $(\sim 0.1 \mathrm{ml})$ of $\mathrm{Fe}\left(\mathrm{NO}_{3}\right)_{3}$ in ethanol $\left(1.2 \times 10^{-3} \mathrm{~mol} / \mathrm{l}\right)$ were placed on the grid. At the substrate temperature of $\sim 100{ }^{\circ} \mathrm{C}$, the ethanol evaporates at a rate that allows the solution to first fill the holes of the grid then evaporate without penetrating into the area shielded by the copper grid to any extent. After several minutes of additional heating to dry up ethanol, the copper grid was lifted off the substrate, leaving a well-defined pattern of catalyst. This substrate was inverted and placed on the top of milled $\mathrm{B}_{4} \mathrm{C}$ powders directly, and annealed at $1300{ }^{\circ} \mathrm{C}$ for $8 \mathrm{~h}$ in a tube furnace with a flow of nitrogen of $\sim 1.41 / \mathrm{min}$. The morphology of the substrate surface was 


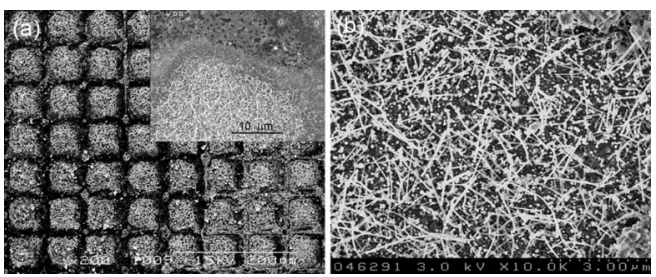

FIG. 1. SEM images of the as-grown conical nanorod deposits: (a) lowmagnification image showing the patterned growth, the inset shows a welldefined boundary between the growth and nongrowth regions, and (b) highmagnification image displaying the typical morphology of the nanorods.

observed with a field emission scanning electron microscope (FESEM) (Hitachi 4500). The structure of the nanorods were studied by using a transmission electron microscope (Philips CM300) working at $300 \mathrm{kV}$. The composition of the nanorods was identified by using an energy-dispersive $\mathrm{X}$-ray spectrometer (EDX), fitted with a superultrathin window. Cathodoluminescence (CL) spectra of the sample were measured by using a JEOL 35C FESEM (the accelerating voltage and beam current was $20 \mathrm{kV}$ and $100 \mathrm{nA}$, respectively) equipped with an Oxford Instruments MonoCL2 and CL spectra were collected at both 300 and $80 \mathrm{~K}$ using a liquid nitrogen cold stage. All CL spectra have been corrected for the response of the collection system. Panchromatic CL images of the pattern were taken by using an off-axis mirror and Hitachi CL detector in a Hitachi S2250-N.

The typical morphology of the as-grown conical boron nitride nanorod deposit is shown in the SEM image of Fig. 1(a). Large quantities of nanorods grew on the $50 \mu \mathrm{m}$ blocks corresponding to the squares of the copper grid, the pattern being produced uniformly in a macroscopic scale of $3 \mathrm{~mm}$. It is expected that patterns with different configurations and larger areas can be achieved by using this simple method. The inset of Fig. 1(a) indicates the well-defined boundaries of the deposits. Figure 1(b) reveals the morphologies of individual nanorods. The lengths of the nanorods can be up to $\sim 10 \mu \mathrm{m}$, and their diameters are around $60 \mathrm{~nm}$ on average, and they are randomly oriented.

Transmission electron microscopy (TEM) analyses reveal more details of the structure of the as-grown nanorods. As shown in Fig. 2(a), the nanorods typically exhibit straight-rod morphology. A tip particle, which is the Fe-Si alloy enclosed by BN layers, can be often observed on one end of a nanorod [black arrows in Fig. 2(a)]. The inset of Fig. 2(a) is a selected-area electron diffraction pattern taken from a single nanorod. Two rows of $000 l$ reflection spots as well as 10.0 reflection loci can be discerned. This indicates that the incident electron beam was more or less perpendicular to the axis of the nanorod and suggests a conical morphology of the nanorod. ${ }^{13,23}$ The apex angle of the nanorod can be estimated by the interangle of the two sets of $000 l$ spots. Lattice image, Fig. 2(b), shows the conical nature of the nanorods clearly. The inclination of the $000 l$ fringes (see the inset) and the nanorod axis is clearly seen. The distribution of the apex angle of the nanorods does not support any nonhexagonal member rings, which, according to some researchers, is evidence of a helical structure. ${ }^{13,23}$ However, as shown in Fig. 2(b), voids exist at the cores of the nanorods alternating with lattice fringes which extend continuously from one side of the nanorod to the other side. The existence of these voids cannot be explained with a helical model, and it is more likely that the nanorods consist of stacked cones.

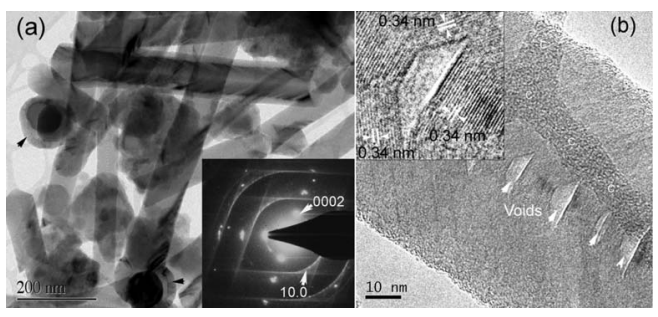

FIG. 2. (a) Bright-field TEM image shows the nanorods have straight rodlike morphology and catalyst particles are indicated by the black arrows; the inset is the selective area electron diffraction pattern from an individual nanorod and two rows of $000 \mathrm{l}$ can be discerned. (b) The detail of one nanorod shows typical conical nature with voids (arrowed) existing in the center of the nanorods, and magnified in the inset to show BN lattice fringes. The speckled feature in the background marked with a series of " $c$ "s is the lacey-carbon film supporting the sample for TEM.

EDX results show that the nanorods are composed of only boron and nitrogen.

Figure 3 are CL spectra of the specimen measured with a scan area of $17 \mu \mathrm{m} \times 14 \mu \mathrm{m}$, and spectra in Fig. 3(a) were collected from the same region of specimen and spectra in Fig. 3(b) were collected from the same region but a different analysis area to Fig. 3(a). All the CL spectra have two broadbands: an ultraviolet band and a red band centered at about $\sim 3.8$ and $\sim 1.8 \mathrm{eV}$, respectively. A band structure calculation taking into account interlayer interaction proposes that $h \mathrm{BN}$ exibits an indirect gap of $3.9 \mathrm{eV}$ and the UV band of the nanorods could be the near-band-edge emission. ${ }^{2,3}$ However, values of $h \mathrm{BN}$ band gap in literature reports have a wide range $e^{24,25}$ and often extend outside the detection range of the CL system used in this work. Consequently, it is possible that the origin of the observed $3.8 \mathrm{eV}$ emission can be attributed to defect-related centers instead of band-to-band transitions. In this case, the red shift of the $3.8 \mathrm{eV}$ peak with increasing specimen temperature could result from the presence of two centers at 3.4 and 3.8 eVeach with a different thermal behavior rather than an increase in the band gap due to lattice expansion. The temperature and excitation density dependence of the $3.8 \mathrm{eV}$ peak [shown in Figs. 3(a) and 3(b), respectively] are consistent with a shallow donor-deep acceptor pair (DAP). The blue shift of the $3.8 \mathrm{eV}$ band with increasing beam current can be attributed to saturation of more distant donor-acceptor pairs while the decrease in $3.8 \mathrm{eV}$ intensity with increasing temperature can be explained by thermal ionization of the shallow donor involved in the DAP. The decrease in the $3.8 \mathrm{eV}$ vs $1.8 \mathrm{eV}$ intensity ratio with increasing beam current [Fig. 3(b)] indicates that the $3.8 \mathrm{eV}$ emission is saturating with respect to the $1.8 \mathrm{eV}$. This result also suggests that the $3.8 \mathrm{eV}$ peak is defect related because saturation behavior is not expected for bandto-band transitions.

The origin of the red band, which has been observed in $c \mathrm{BN}$ and boron nitride whiskers, is also controversial. ${ }^{15}$ For the $c \mathrm{BN}$, vacancy-type defect and dislocation bands are responsible for the emission, while $s p^{3}$-bonded structures and size confinement have been considered in the whisker case. As shown in Fig. 2(b), to form the cup-like morphology, atomic configurations in the nanorods must have a number of nonhexagonal rings around the voids where vacancy-type defect and dislocation may well be introduced. On the surfaces of the nanorods, the BN cones within each stack are interconnected through more or less $s p^{3}$-hybridized bonds. Considering the small dimensions and thus the large surface-to- 

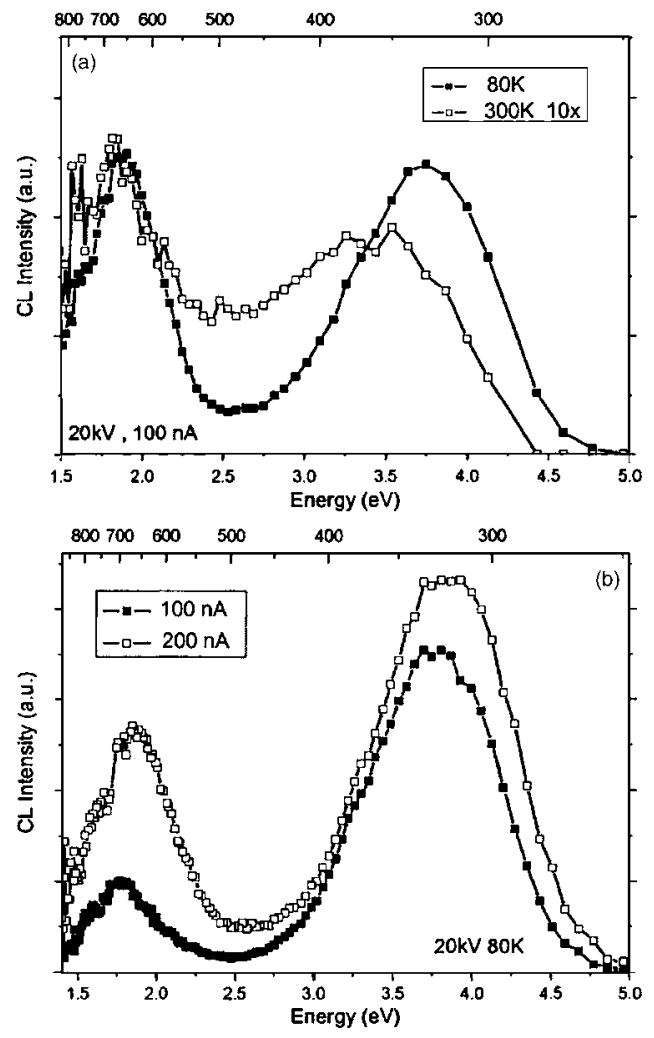

FIG. 3. Cathodoluminescence spectra taken at (a) 300 and $80 \mathrm{~K}$; (b) different excitation powers: 100 and $200 \mathrm{nA}$.

volume ratios of the nanorods, we believe the red band of our sample may also arise from $s p^{3}$-bonded structures and size confinement. Nevertheless, given the wide band gap of boron nitride, the red luminescence band (RL) is a deep-level feature. The very small blueshift of the RL with decreasing temperature from 300 to $80 \mathrm{~K}$ indicates that deep level centers are indeed involved. The blueshift of the RL emission under high excitation energy emission indicates that the RL emission has a DAP character. Finally, panchromatic CL images are shown in Fig. 4. Figure 4(a) clearly displays the overall pattern and Fig. 4(b) shows enhanced emission near the catalyst particle region. TEM analysis shows that BN layers enclose these particles and the catalyst particles of iron silicide are metallic in nature due to their high $\mathrm{Fe}$ ratios (>80 at. \%). ${ }^{26}$ Hence, it is safe to claim that the emission near these particles originates from the surrounding BN layers. Although these BN layers have no essential structural difference from the nanorod body (i.e., they are both basically $h \mathrm{BN}$ ), they might contain more defects and dangling bonds because of their large curvatures, which could be responsible for some of the CL peaks. In addition, enhanced emission from Fe-contaminated $\mathrm{BN}$ has been observed by several authors for some emission bands,${ }^{7,8}$ while in our case it is hard to verify that $\mathrm{Fe}$ atoms are incorporated into the BN lattice. Further experimental and theoretical work is required to clarify the relationship between the emission, the structure of the nanorods, and the catalyst particles.

In conclusion, a simple and effective approach for growing large-scale, high-density, and well-patterned conical boron nitride nanorods has been realized. The as-grown nanorods exhibit uniform morphology and the catalyst pattern precisely defines the locations of nanorod deposition. The nanorods consist of stacked cones with voids existing in their

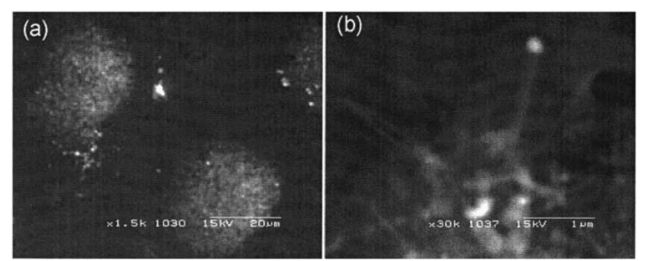

FIG. 4. Panchromatic CL images (a) showing the square pattern structure inclined $\sim 40^{\circ}$ to the image edges (b) strong emission from the catalyst region.

core region. Two emission bands centered at $\sim 3.8$ and $\sim 1.8 \mathrm{eV}$ were observed in their cathodoluminescence spectra. CL images display clear pattern structures. The selective growth and the strong CL emission of the nanorods suggest promising UV applications of the nanorods.

The authors appreciate Dr. Sally Stowe for helpful discussions and assistance with the SEM and CL, and they thank Dr. Cheng Huang for assistance with the SEM. This work is financially supported, in part, by The Australian Research Council under the nanotube program of the Center for Functional Nanomaterials.

${ }^{1}$ A. V. Kurdyumov, V. L. Solozhenko, and W. B. Zelyavski, J. Appl. Crystallogr. 28, 540 (1995).

${ }^{2}$ A. Catellani, M. Posternak, and A. Baldereschi, Phys. Rev. B 32, 6997 (1985).

${ }^{3}$ W. J. Zhang, H. Kanda, and S. Matsumoto, Appl. Phys. Lett. 81, 3356 (2002).

${ }^{4}$ R. F. Davis, Proc. IEEE 79, 702 (1991).

${ }^{5}$ A. Zunger, A. Katzir, and A. Halperin, Phys. Rev. B 13, 5560 (1976).

${ }^{6}$ D. M. Hoffman, G. L. Doll, and P. C. Eklund, Phys. Rev. B 30, 6051 (1984).

${ }^{7}$ B. Yao, L. Liu, W. H. Su, Z. X. Shen, L. Liu, W. X. Sun, and J. Ding, J. Appl. Phys. 96, 1947 (2004).

${ }^{8}$ B. Yao, Z. X. Shen, L. Liu, and W. H. Su, J. Phys.: Condens. Matter 16, 2181 (2004).

${ }^{9}$ C. A. Taylor, S. W. Brown, V. Subramaniam, S. Kidner, S. C. Rand, and R. Clarke, Appl. Phys. Lett. 65, 1251 (1994).

${ }^{10}$ O. Mishima, K. Era, J. Tanaka, and S. Yamaoka, Appl. Phys. Lett. 53, 962 (1988).

${ }^{11}$ K. Watanabe, T. Taniguchi, and H. Kanda, Phys. Status Solidi A 201, 2561 (2004).

${ }^{12}$ N. G. Chopra, R. J. Luyken, K. Cherrey, V. H. Crespi, M. L. Cohen, S. G. Louie, and A. Zettl, Science 269, 966 (1995).

${ }^{13}$ L. Bourgeois, Y. Bando, S. Shinozaki, K. Kurashima, and T. Sato, Acta Crystallogr., Sect. A: Found. Crystallogr. A55, 168 (1999).

${ }^{14}$ F. F. Xu and Y. Bando, Acta Crystallogr., Sect. A: Found. Crystallogr. A59, 168 (2003).

${ }^{15}$ Y. C. Zhu, Y. Bando, D. F. Xue, T. Sekiguchi, D. Golberg, F. F. Xu, and Q. L. Liu, J. Phys. Chem. B 108, 6193 (2004).

${ }^{16}$ X. Blase, A. Rubio, S. G. Louie, and M. L. Cohen, Europhys. Lett. 28, 335 (1994).

${ }^{17}$ L. Brus, J. Phys. Chem. 90, 2555 (1986).

${ }^{18}$ T. Takagahara and K. Takeda, Phys. Rev. B 46, 15578 (1992).

${ }^{19}$ T. Sekiguchi, Q. Liu, T. Tanaka, J. Hu, Y. Zhu, and Y. Bando, Eur. Phys. J.: Appl. Phys. 27, 107 (2004).

${ }^{20}$ H. Z. Zhang, J. D. Fitz Gerald, J. Yu, and Y. Chen, J. Am. Ceram. Soc. 89, 675 (2006).

${ }^{21}$ Y. Chen, J. D. Fitz Gerald, J. S. Williams, and S. Bulcock, Chem. Phys. Lett. 299, 260 (1999).

${ }^{22}$ Y. Chen, L. T. Chadderton, J. Fitz Gerald, and J. S. Williams, Appl. Phys. Lett. 74, 2960 (1999).

${ }^{23}$ L. Bourgeois, Y. Bando, K. Kurashima, and T. Sato, Philos. Mag. A 80, 129 (2000).

${ }^{24}$ C. Y. Zhi, Y. Bando, C. C. Tang, D. Golberg, R. G. Xie, and T. Sekiguchi, Appl. Phys. Lett. 86, 213110 (2005).

${ }^{25}$ K. Watanabe, T. Taniguchi, and H. Kanda, Nat. Mater. 3, 404 (2004).

${ }^{26}$ S. P Murarka, Annu. Rev. Mater. Sci. 13. 117 (1983). 